\title{
Correlation between myocardial enzyme serum levels and markers of inflammation with severity of coronary artery disease and Gensini score: A hospital-based, prospective study in Greek patients
}

\author{
Vasileios Peppes \\ George Rammos \\ Efstathios Manios \\ Eleni Koroboki \\ Stylianos Rokas \\ Nikolaos Zakopoulos \\ Department of Clinical Therapeutics, \\ Alexandra Hospital, University \\ of Athens School of Medicine, \\ Athens, Greece
}

Background: Our objective was to associate serum levels of myocardial enzymes and inflammatory biomarkers with severity of coronary artery disease (CAD).

Patients and methods: 123 patients participated in our study, including 65 cases of acute myocardial infarction (MI), 27 cases of newly diagnosed CAD - without MI - and 31 controls. In all subjects, myocardial serum enzyme levels (creatine phosphokinase, aspartate aminotransferase, lactate dehydrogenase) and inflammatory indices (C-reactive protein, fibrinogen, white blood cells, and erythrocyte sedimentation rate) were measured. Patients were all submitted to coronary angiography and CAD severity was evaluated by Gensini score.

Results: Significant differences concerning enzyme serum levels and inflammatory indices were found to exist between the three study groups, being highest among patients with acute MI $(\mathrm{p}<0.001)$. A significant association was demonstrated between Gensini score and serum enzyme levels as well as inflammatory biomarkers.

Conclusions: Our findings suggest that serum levels of myocardial enzymes and inflammatory indices correlate with CAD severity in Greek patients.

Keywords: myocardial enzymes, inflammation, coronary angiography, coronary artery disease

\section{Background}

Acute coronary syndromes including ST-elevation myocardial infarction (STEMI) and Non-ST elevation myocardial infarction (NSTEMI) are known to be associated with elevated serum levels of myocardial enzymes, including troponin, total creatine phospokinase (CPK), the myocardial isoenzyme of CPK (CPK-MB), aspartate aminotransferase (AST) and lactate dehydrogenase (LDH). Unstable angina pectoris is also included in acute coronary syndromes as a harbinger of imminent myocardial necrosis in the setting of either STEMI or NSTEMI. Myocardial enzyme levels are used in both clinical and laboratory settings in order to establish a diagnosis of an acute coronary syndrome, apart from electrocardiographic and clinical findings (Möckel et al 1998; Panteghini 2004; Antman et al 2004; Melanson et al 2007; Morrow et al 2007).

The inflammatory process initiated by myocardial tissue necrosis (Neumann et al $1995)$ is also related to an increase of nonspecific serum markers such as C-reactive protein (CRP) (Mendall et al 2000; Blake and Ridker 2003; Brunetti et al 2006), fibrinogen (Eidelman and Hennekens 2003; Manilla 2006; Omran and Asadollahi 2007), peripheral white blood cell count (WBC) (Madjid et al 2004; Jia et al 2005), and erythrocyte sedimentation rate (ESR) (Erikssen et al 2000) above normal limits. 
Severity of coronary artery disease (CAD) is optimally assessed by means of coronary angiography where the number of epicardial coronary vessels affected, the anatomical distribution of critical stenotic lesions and the extent of vascular flow impairment is demonstrated (Conti 1977; Gensini 1980). Coronary angiography remains the standard for assessment of anatomic coronary disease, because no other currently available test can accurately define the extent of coronary luminal obstruction. Coronary angiography is principally used in three clinical situations: (i) to determine the presence and extent of obstructive CAD in a setting in which the diagnosis is uncertain and CAD cannot be reasonably excluded by noninvasive testing; (ii) to assess the feasibility and appropriateness of various forms of therapy, such as revascularization by percutaneous or surgical interventions; and (iii) as a research tool for the assessment of treatment results and the progression or regression of coronary atherosclerosis (Scanlon et al 1999). The Gensini scoring system is a valuable aid, used to estimate the gravity of CAD according to angiographic findings, taking into account the anatomical location and the degree of coronary vessel stenoses. Gensini score calculation is based on the evaluation of the number of stenotic coronary artery segments, the degree of their lumen stenosis and the localization of stenotic changes (Gensini 1983).

Limited information is available regarding the correlation between myocardial enzyme serum levels and inflammatory markers with the extent and severity of CAD, as it is estimated by Gensini score in Greek patients submitted to coronary angiography. A prospective, observational, case control study was performed in a total of 123 hospitalized patients at the
Department of Clinical Therapeutics, Alexandra Hospital between September 2005 and September 2007. The objective was to identify correlations between myocardial enzyme serum levels, inflammatory biomarkers, and coronary angiography findings, represented by Gensini score.

\section{Patients and methods}

\section{Study population}

Among the study population, there were 65 cases of first-ever acute myocardial infarction (MI) (STEMI and NSTEMI), and 27 cases of newly-diagnosed CAD without evidence of MI in the past or during hospitalization. There were also 31 cases with negative angiographic findings that comprised the control group of patients. Cases of acute, first-ever MI included 42 male patients (34.1\% of total study population) with mean age of $60 \pm 10.9$ years. Cases of newly-diagnosed CAD without MI included 17 male patients (13.18\% of total study population) with mean age of $64 \pm 11$ years. Finally, subjects with negative coronary angiography (control group) included 7 male patients (5.6\% of total study population) with mean age of $64.3 \pm 10.5$ years (Table 1). Cases in all three patient subgroups were matched for age, body mass index (BMI), and waist-to-hip ratio (WHR) (Table 1).

In order to perform our study, a convenience sample of patients sequentially hospitalized between September 2005 and September 2007 that were submitted to coronary angiography, was selected. Patients in the control group were submitted to coronary angiography as part of a detailed diagnostic workup for a variety of conditions. The main reasons of hospitalization for controls were either atypical chest pain with ambiguous exercise stress testing

Table I Baseline characteristics of the study population. All three patient subgroups are matched for age, BMI, and WHR. There was a significant difference in the prevalence of male sex, smoking habits, arterial hypertension, hyperlipidemia, and family history of CAD between patient subgroups (see details in text)

\begin{tabular}{|c|c|c|c|c|}
\hline & $\begin{array}{l}\text { Acute first-ever myocardial } \\
\text { infarction (STEMI \& NSTEMI) }\end{array}$ & $\begin{array}{l}\text { Coronary artery disease } \\
\text { (no myocardial infarction) }\end{array}$ & Controls & $\mathbf{p}$ \\
\hline$N$ & 65 & 27 & 31 & \\
\hline Male & $42(34 . I)^{*}$ & $17(13.8)$ & $7(5.6)$ & $<0.001$ \\
\hline Mean age (Years) & $60 \pm 10.9$ & $64 \pm 11$ & $64.3 \pm 10.5$ & 0.113 \\
\hline Smoking & $38(30.8)$ & $15(12.2)$ & $10(8.1)$ & 0.049 \\
\hline Hypertension & $4 \mathrm{I}(33.3)$ & $23(18.7)$ & $25(20.3)$ & 0.048 \\
\hline Diabetes mellitus & $12(9.7)$ & $10(8.1)$ & $6(4.8)$ & 0.134 \\
\hline Hyperlipidemia & $60(48.7)$ & $26(2 I . I)$ & $19(15.4)$ & $<0.001$ \\
\hline Family History of CAD & $23(18.7)$ & $2(1.6)$ & $9(7.6)$ & 0.023 \\
\hline Alcohol consumption & $4(3.2)$ & $2(1.6)$ & $\mathrm{I}(0.8)$ & 0.769 \\
\hline Hyperuricemia & $18(14.6)$ & $7(5.6)$ & $6(4.8)$ & 0.676 \\
\hline
\end{tabular}

*Numbers in brackets indicate corresponding percentages of the total study population. 
results $(\mathrm{N}=18,58 \%)$, or presurgical evaluation for severe valvular disorders - aortic stenosis, mitral stenosis, mitral incompetence $-(\mathrm{N}=6,19.4 \%)$, dilated or hypertrophic cardiomyopathy $(\mathrm{N}=3,9.7 \%)$, and nonsustained ventricular tachycardia $(\mathrm{N}=1,3.2 \%)$.

\section{Methods}

A detailed medical history, physical examination, electrocardiogram, hematologic and biochemical screening was performed in all patients during hospitalization. In all three groups of patients, laboratory evaluation of total CPK, AST, LDH, CRP, fibrinogen, WBC count, and ESR was performed in the first $48 \mathrm{~h}$ during hospital stay. Although serum troponin I test was qualitatively evaluated and regarded as either "positive", indicating increased serum levels or "negative", assuming normal serum levels, no further quantitative determination of troponin I was performed due to laboratory limitations. Serial biochemical and hematological assays were performed, yet peak serum levels of myocardial enzymes and inflammation markers were utilized in our analyses.

All patients were submitted to coronary angiography during hospitalization, either on an emergency basis or as part of a scheduled diagnostic workup. Patients in all study subgroups had no underlying systemic disease that could affect serum inflammatory biomarker profile apart from cardiological conditions. Consequently, all measurements reflect the impact of CAD upon enzyme and inflammatory biomarker levels.

The Gensini scoring system was utilized in the evaluation of CAD severity. The Gensini score was calculated for each patient from the coronary arteriogram by assigning a severity score to each coronary stenosis according to the degree of luminal narrowing and its geographic importance. Reduction in the lumen diameter, and the roentgenographic appearance of concentric lesions and eccentric plaques were evaluated (reductions of $25 \%, 50 \%, 75 \%, 90 \%, 99 \%$, and complete occlusion were given Gensini scores of 1, 2, 4, 8, 16, and 32, respectively). Each principal vascular segment was assigned a multiplier in accordance with the functional significance of the myocardial area supplied by that segment: the left main coronary artery $\times 5$; the proximal segment of left anterior descending coronary artery $(\mathrm{LAD}) \times 2.5$; the proximal segment of the circumflex artery $\times 2.5$; the mid-segment of the $\mathrm{LAD} \times 1.5$; the right coronary artery, the distal segment of the LAD, the posterolateral artery and the obtuse marginal artery $\times 1$; and others $\times 0.5$. Scoring was performed by two observers and averaged (Hayashi et al 2003).

\section{Assesment of risk factors and comorbidities}

A positive family history was sought in all patients with a first degree relative with evidence of CAD. Smoking habits and alcohol consumption was also recorded. Hypertensive patients were identified in the basis of receiving antihypertensive treatment before hospital admission or whether blood pressure exceeded 140/90 $\mathrm{mmHg}$ in at least three measurements. Patients with diabetes mellitus were identified in the basis of receiving oral hypoglycemic medications or insulin, or having fasting glucose levels above $126 \mathrm{mg} / \mathrm{dl}$. Hyperlipidemia was identified in patients already on hypolipidemic treatment, or in patients with acute MI or CAD (without infarction) or diabetes mellitus with low-density lipoprotein $>100 \mathrm{mg} / \mathrm{dl}$. Patients with triglyceride levels above $150 \mathrm{mg} / \mathrm{dl}$ were also regarded as being hyperlipidemic.

Baseline characteristics of the study population are demonstrated in Table 1.

\section{Statistical analysis}

Chi-square test was performed to detect differences in the prevalence of classic cardiovascular risk factors (smoking, hypertension, diabetes mellitus, hyperlipidemia, family history of CAD) between the three patient groups. Analysis of variance (ANOVA) was performed in order to identify differences concerning mean serum enzyme levels and inflammatory biomarkers between patient groups. Bivariate correlation analysis was conducted in order to detect correlation of myocardial enzyme serum levels and inflammatory markers with Gensini score. SPSS 13.0 statistical software (SPSS Inc., Chicago, IL) was used in the analysis performed.

\section{Results}

A statistically significant difference in the prevalence of male sex, smoking habits, hypertension, hyperlipidemia, and family history of CAD was found to exist among the three patient groups. In particular, male sex predominated in patients with acute first-ever MI $(\mathrm{N}=42)$ and CAD without MI $(\mathrm{N}=17)$ in comparison with the control group $(\mathrm{N}=7)$, ( $\mathrm{p}<0.001)$, (Table 1).

No significant differences between patient groups regarding mean age, BMI and WHR were observed. In particular, patients with acute, first-ever MI had mean age $60 \pm 10.9$ years whereas patients with CAD without MI had mean age $64 \pm 11$ years and control subjects had mean age $64,3 \pm 10.5$ years, respectively $(\mathrm{p}=0.113,95 \% \mathrm{CI})($ Table 1$)$. Patients with acute, first-ever MI had mean BMI values of 
$27.4 \pm 3.69 \mathrm{~kg} / \mathrm{m}^{2}$ and mean WHR values of $0.95 \pm 0.08$ while patients with $\mathrm{CAD}$ without MI had mean BMI values of $27.7 \pm 5.24 \mathrm{~kg} / \mathrm{m}^{2}$ and mean WHR values of $0.93 \pm 0.07$. Control subjects had mean BMI $29.2 \pm 5 \mathrm{~kg} / \mathrm{m}^{2}$ and mean WHR $1.07 \pm 0.97$. No significant differences regarding BMI $(p=0.219,95 \% \mathrm{CI})$ or WHR $(p=0.511,95 \% \mathrm{CI})$ were found between patient subgroups (Table 1).

The prevalence of tobacco use was significantly higher in patients with acute first-ever $\mathrm{MI}(\mathrm{N}=38)$ and CAD without MI $(\mathrm{N}=17)$ compared with the controls $(\mathrm{N}=7)$, $(p=0.049)$ (Table 1). Similarly, the prevalence of hypertension, hyperlipidemia and positive family history of CAD was found to be higher in patients with acute MI and CAD without MI, in contrast with controls ( $p=0.048, p<0.001$ and $\mathrm{p}=0.023$ respectively; Table 1 ). Diabetes mellitus and hyperuricemia were observed in higher proportion of patients with acute $\mathrm{MI}$ and $\mathrm{CAD}$ without $\mathrm{MI}$, in contrast with controls, yet the difference did not achieve statistical significance in our study sample. ( $p=0.13$ and $p=0.67$ respectively; Table 1). Baseline characteristics of the study population are shown in Table 1.

Mean levels of CPK, AST, and LDH were significantly higher in patients with acute first-ever MI compared with patients with CAD without MI and controls. Mean serum CPK value was in the range of $1.241 \pm 1.459 \mathrm{IU} / \mathrm{L}$ for patients with acute MI, $59 \pm 21 \mathrm{IU} / \mathrm{L}$ for patients with $\mathrm{CAD}$ without MI and $62 \pm 30 \mathrm{IU} / \mathrm{L}$ for controls $(\mathrm{p}<0.001,95 \%$ CI; Table 2). Similarly, mean serum AST was $154 \pm 141 \mathrm{IU} / \mathrm{L}$ and mean serum LDH was $610 \pm 523 \mathrm{IU} / \mathrm{L}$ in patients with acute MI, while patients with CAD but no MI had mean levels of AST $20 \pm 8 \mathrm{IU} / \mathrm{L}$ and LDH $188 \pm 67 \mathrm{IU} / \mathrm{L}$. Control patients had mean serum AST $19 \pm 7$ IU/L and LDH $193 \pm 44$ IU/L. ( $\mathrm{p}<0.001$ and $\mathrm{p}<0.001$ respectively, 95\% CI, Table 2). Apparently, patients with acute MI had significantly higher levels of CPK, AST, and LDH compared to patients in the other two groups (Table 2).

Mean values of CRP and peripheral WBC counts were significantly higher in patients with acute MI than in patients with CAD without $\mathrm{MI}$ and controls. In particular, mean CRP levels were $4.7 \pm 5.5 \mathrm{mg} / \mathrm{dl}, 0.8 \pm 0.6 \mathrm{mg} / \mathrm{dl}$, and $0.77 \pm 0.5 \mathrm{mg} / \mathrm{dl}$, respectively ( $<<0.001$; Table 2 ). Mean peripheral WBC counts were significantly higher in patients with acute $\mathrm{MI}\left(11.216 \pm 3.496 / \mathrm{mm}^{3}\right)$ compared with patients with CAD without $\mathrm{MI}\left(8.162 \pm 2.456 / \mathrm{mm}^{3}\right)$ and controls $\left(7.648 \pm 1.903 / \mathrm{mm}^{3}\right),(\mathrm{p}<0.001$; Table 2$)$. Fibrinogen levels and ESR were higher in patients with acute MI compared to other groups, yet the difference did not yield statistical significance in our study sample $(p=0.14$ and $p=0.33$, respectively; Table 2). Regarding serum lipid profile, although mean HDL levels were higher in controls $(64 \pm 33 \mathrm{mg} / \mathrm{dl})$ in comparison with patients with CAD without MI $(48 \pm 11 \mathrm{mg} / \mathrm{dl})$ and patients with acute MI $(55 \pm 29 \mathrm{mg} / \mathrm{dl})$, the difference

Table 2 Mean values \pm SD of biochemical, hematological parameters and Gensini score of patient subgroups. Peak serum myocardial enzymes (total CPK, AST, and LDH), inflammatory markers (CRP, peripheral blood WBC) and Gensini score were significantly higher in patients with acute, first-ever MI

\begin{tabular}{|c|c|c|c|c|}
\hline & $\begin{array}{l}\text { Acute first-ever } \\
\text { myocardial infarction } \\
\text { (STEMI \& NSTEMI) }\end{array}$ & $\begin{array}{l}\text { Coronary artery disease } \\
\text { (no myocardial infarction) }\end{array}$ & Controls & $\mathbf{p}$ \\
\hline $\mathrm{Hb}(\mathrm{g} / \mathrm{dl})$ & $13.6 \pm 1.44$ & $13.3 \pm 1.54$ & $12.93 \pm 1.56$ & 0.12 \\
\hline $\mathrm{PLT}(/ \mu \mathrm{L})$ & $246.531 \pm 102.879$ & $213.920 \pm 41.819$ & $251.933 \pm 58.215$ & 0.18 \\
\hline WBC $(/ \mu \mathrm{L})$ & $11.216 \pm 3.496$ & $8.162 \pm 2.456$ & $7.648 \pm 1.903$ & $<0.001$ \\
\hline CPK (IU/L) & $1241.6 \pm 1.459$ & $59.4 \pm 21$ & $62.8 \pm 30.6$ & $<0.001$ \\
\hline AST (IU/L) & $154.2 \pm|4| .7$ & $20.1 \pm 8.3$ & $19.7 \pm 7$ & $<0.001$ \\
\hline LDH (IU/L) & $610.6 \pm 513.4$ & $188.6 \pm 67.8$ & $193.5 \pm 44.8$ & $<0.001$ \\
\hline CRP $(\mathrm{mg} / \mathrm{dl})$ & $4.77 \pm 5.54$ & $0.88 \pm 0.6$ & $0.77 \pm 0.5$ & $<0.001$ \\
\hline $\mathrm{ESR}(\mathrm{mm})$ & $39.9 \pm 28.7$ & $30.2 \pm 25.9$ & $31.8 \pm 20.9$ & 0.33 \\
\hline Fibrinogen (mg/dl) & $434 . I \pm|6|$ & $374.6 \pm 96$ & $371 \pm 139.3$ & 0.14 \\
\hline Total Cholesterol (mg/dl) & $207 \pm 43$ & $200 \pm 36$ & $207 \pm 33$ & 0.75 \\
\hline $\mathrm{HDL}(\mathrm{mg} / \mathrm{dl})$ & $55 \pm 29$ & $48 \pm 11$ & $64 \pm 33$ & 0.13 \\
\hline $\mathrm{LDL}(\mathrm{mg} / \mathrm{dl})$ & $140 \pm 58$ & $|30 \pm 3|$ & $129 \pm 30$ & 0.5 \\
\hline Triglycerides (mg/dl) & $147 \pm 79$ & $158 \pm 101$ & $136 \pm 99$ & 0.68 \\
\hline Gensini Score & $37.4 \pm 31.5$ & $36.67 \pm 35$ & $0.5 \pm 1.3$ & $<0.001$ \\
\hline
\end{tabular}


did not achieve statistical significance in our study population ( $p=0.13$; Table 2). Similarly, mean LDL levels were highest in patients with acute $\mathrm{MI}(140 \pm 58 \mathrm{mg} / \mathrm{dl})$, in contrast with patients with CAD without MI $(130 \pm 31 \mathrm{mg} / \mathrm{dl})$ and controls $(129 \pm 30 \mathrm{mg} / \mathrm{dl})$, but no statistically significant difference was noted in our study sample (Table 2). Mean Gensini score was higher in patients with acute MI $(37.4 \pm 31.5)$ than in patients with CAD without MI $(36.67 \pm 35)$ and controls $(0.5 \pm 1.3)$, ( $\mathrm{p}<0.001,95 \%$ CI; Table 2$)$.

No significant differences regarding cardiac enzymes, inflammatory biomarkers, or Gensini score were noted between patients with STEMI and NSTEMI consequently allowing their incorporation in the same study subgroup under the general term "acute first-ever myocardial infarction."

Bivariate correlation analysis was performed in the whole study sample concerning myocardial enzyme levels (CPK, AST, LDH) and inflammatory indices (CRP, fibrinogen, WBC count, ESR) with Gensini score, in order to establish a possible quantitative association of the latter with severity of CAD. In particular, significant positive
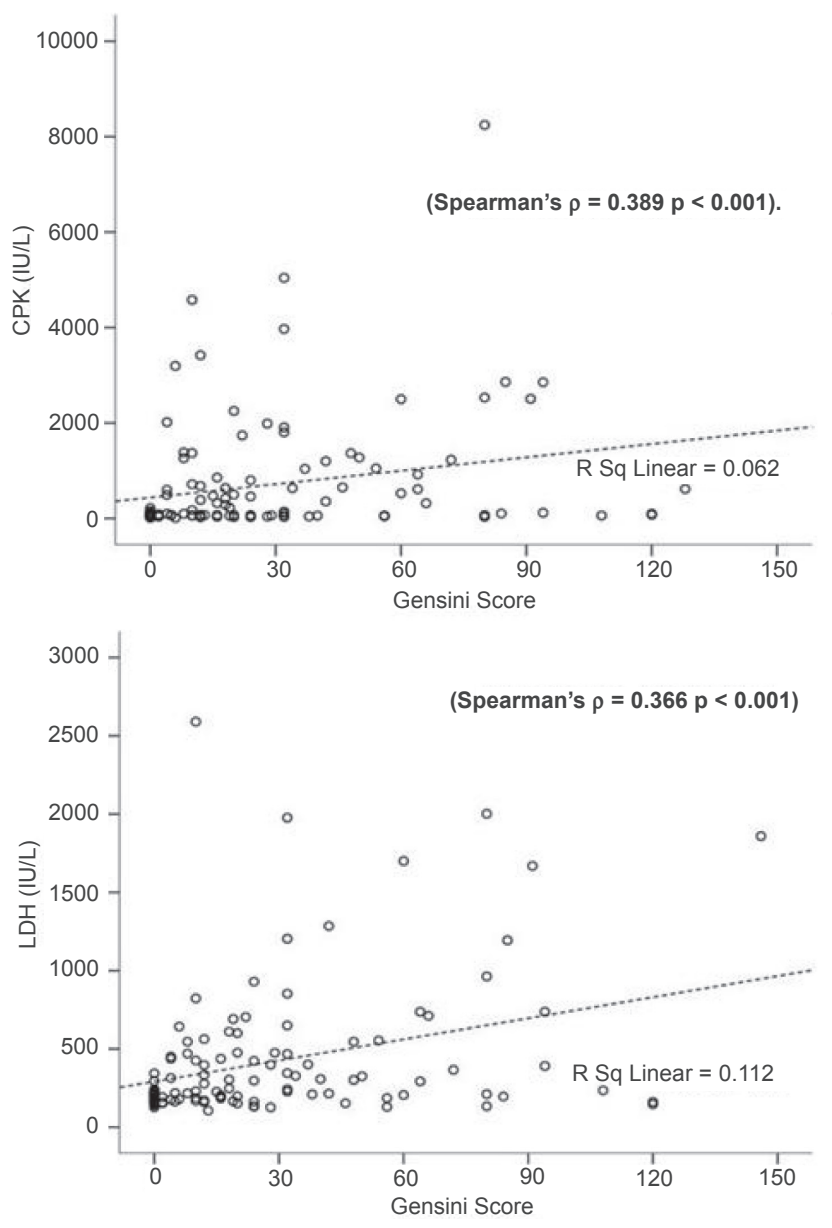

linear between CPK and Gensini score was demonstrated (Spearman's $\rho=0.389$, correlation coefficient $=0.389$, $\mathrm{p}<0.001)$. The same positive correlation was for AST and LDH with Gensini score (Spearman's $\rho=0.391$, correlation coefficient $=0.391, \mathrm{p}<0.001$, and Spearman's $\rho=0.366$, correlation coefficient $=0.366, \mathrm{p}<0.001$, respectively). Therefore, higher myocardial enzyme levels were related to increased severity of CAD, according to Gensini score (Figure 1). Serum HDL levels were inversely correlated with both Gensini score (Spearman's $\rho=-0.205$, correlation coefficient $=-0.205, \mathrm{p}=0.031$ ) and CRP levels (Spearman's $\rho=-0.294$, correlation coefficient $=-0.294$, $\mathrm{p}=0.004$; Figure 2). Therefore, a reciprocal relation between high-density lipoprotein (HDL) levels and severity of CAD (Gensini score) and between serum HDL and CRP levels was observed (Figure 2). Significant correlation was also found regarding CRP and fibrinogen levels with Gensini score (Spearman's $\rho=0.418$, correlation coefficient $=0.418, \mathrm{p}<0.001$ and Pearson correlation $=0.224$, correlation coefficient $=0.143 \mathrm{p}=0.037$, respectively).

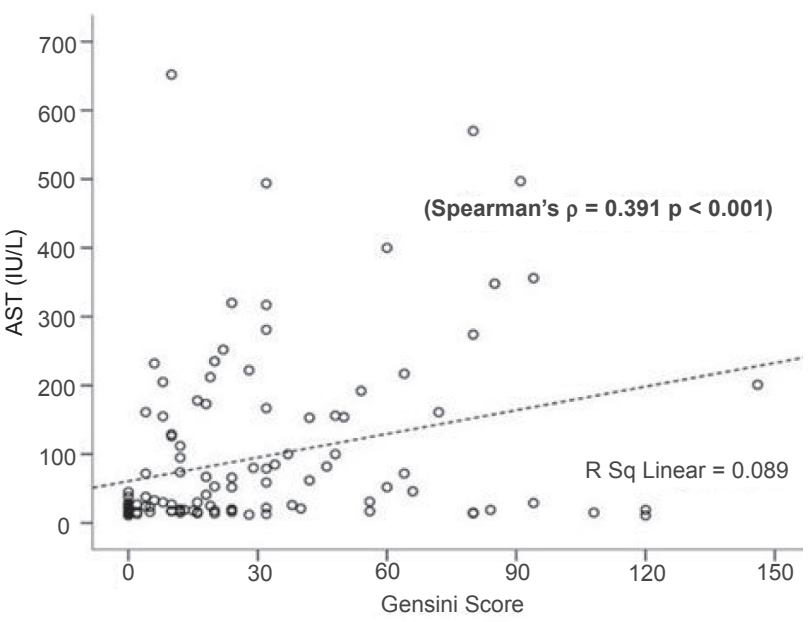

Figure I Scatter plots of peak serum CPK,AST, and LDH (IU/L) with Gensini Score in the whole study sample. Significant positive correlations between peak serum myocardial enzyme levels with Gensini score were identified. 

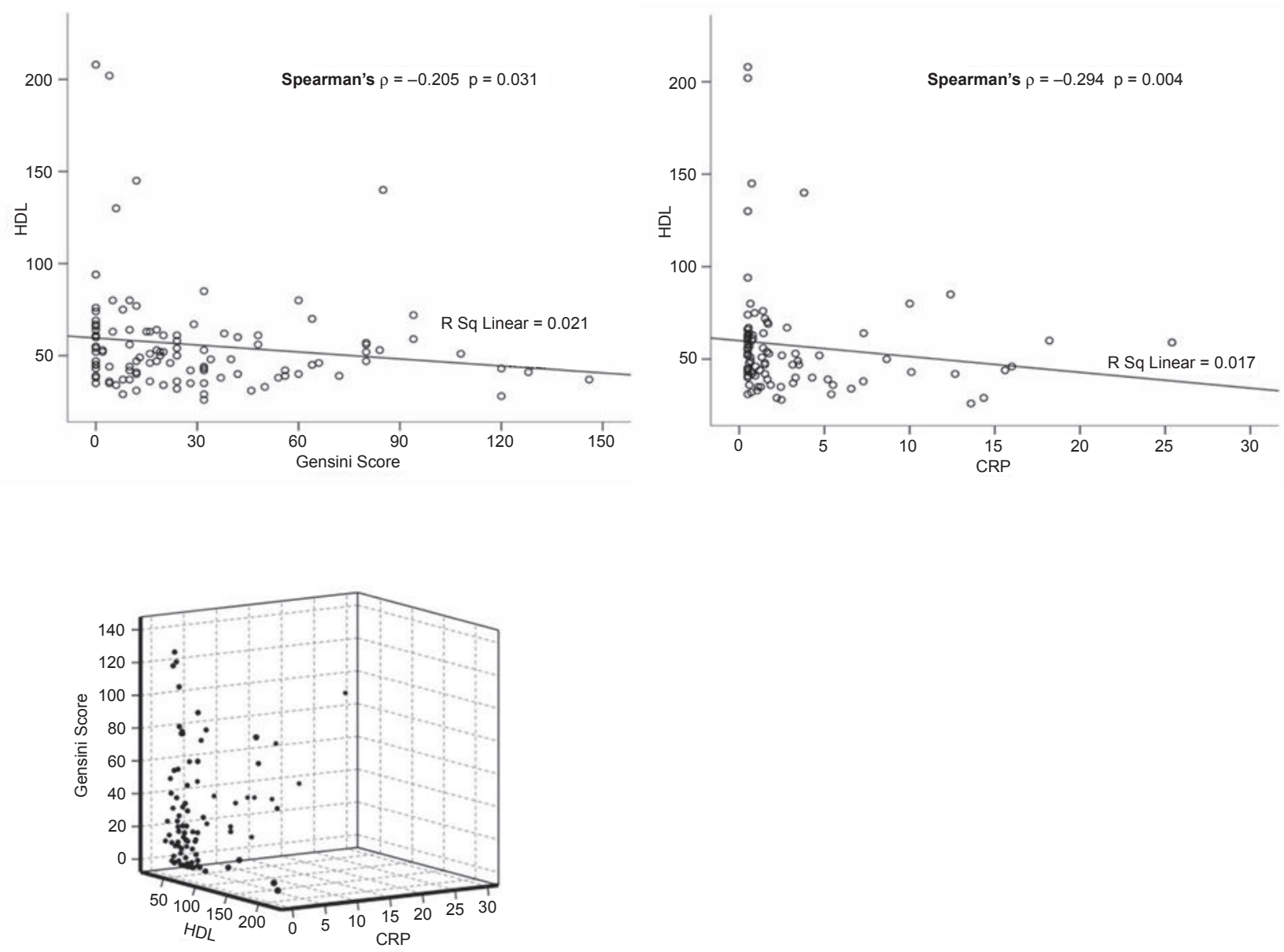

Figure 2 Scatter plots of serum HDL $(\mathrm{mg} / \mathrm{dl})$ with Gensini Score and serum HDL $(\mathrm{mg} / \mathrm{d})$ with peak CRP levels $(\mathrm{mg} / \mathrm{dl})$ in the whole study sample. Significant negative correlations between serum HDL, Gensini score, and peak serum CRP levels were identified.

The same seemed to apply for WBC count and ESR with Gensini score (Spearman's $\rho=0.258$, correlation coefficient $=0.258, p=0.004$ and Spearman's $\rho=0.270$, correlation coefficient $=0.270, \mathrm{p}=0.015$, respectively; Figure 3).

In addition, the latter correlations seemed to apply for each study subgroup of patients. In particular in patients with acute first-ever MI a positive association between both total serum CPK and CRP with Gensini score was identified (correlation coefficients 0.156 and 0.291 , respectively) while a reciprocal association between serum HDL and Gensini score was shown (correlation coefficient $=-0.76$ ) (Figure 4). In patients with newly-diagnosed $\mathrm{CAD}$ without MI positive correlations between total serum CPK and CRP with Gensini score were demonstrated (correlation coefficients $=0.129$ and 0.187 , respectively) and a reciprocal association between serum HDL and Gensini score was also noted (correlation coefficient $=-0.22)($ Figure 5).

\section{Discussion}

In our study, a statistically significant difference in the prevalence of male sex was observed between the three study groups, being higher among patients with acute, first-ever MI and CAD without MI, in comparison with controls $(\mathrm{p}<0.001)$. Male sex is a well recognized risk factor for coronary atherosclerosis since there is a significantly lower age-specific risk of CAD in women than men (Mosca et al 1997).

Our study revealed an increased prevalence of tobacco smoking among patients with acute, first-ever MI and CAD without MI, in comparison with controls $(p=0.049)$. These findings are in accordance with previous large population-based epidemiological studies. In the Western Collaborative Group Study, the risk of CAD was significantly associated both with current and former cigarette usage. The incidence of new clinical coronary heart disease (CHD) was strongly associated with smoking history and with the number of cigarettes smoked (Jenkins et al 1968). In a study of Japanese patients (Yasue et al 2006) low-grade inflammation, atherogenic dyslipidemia, and hypercoagulability were present in smokers compared with those who have never smoked among subjects without apparent inflammation who underwent coronary angiography on suspicion of CAD. 

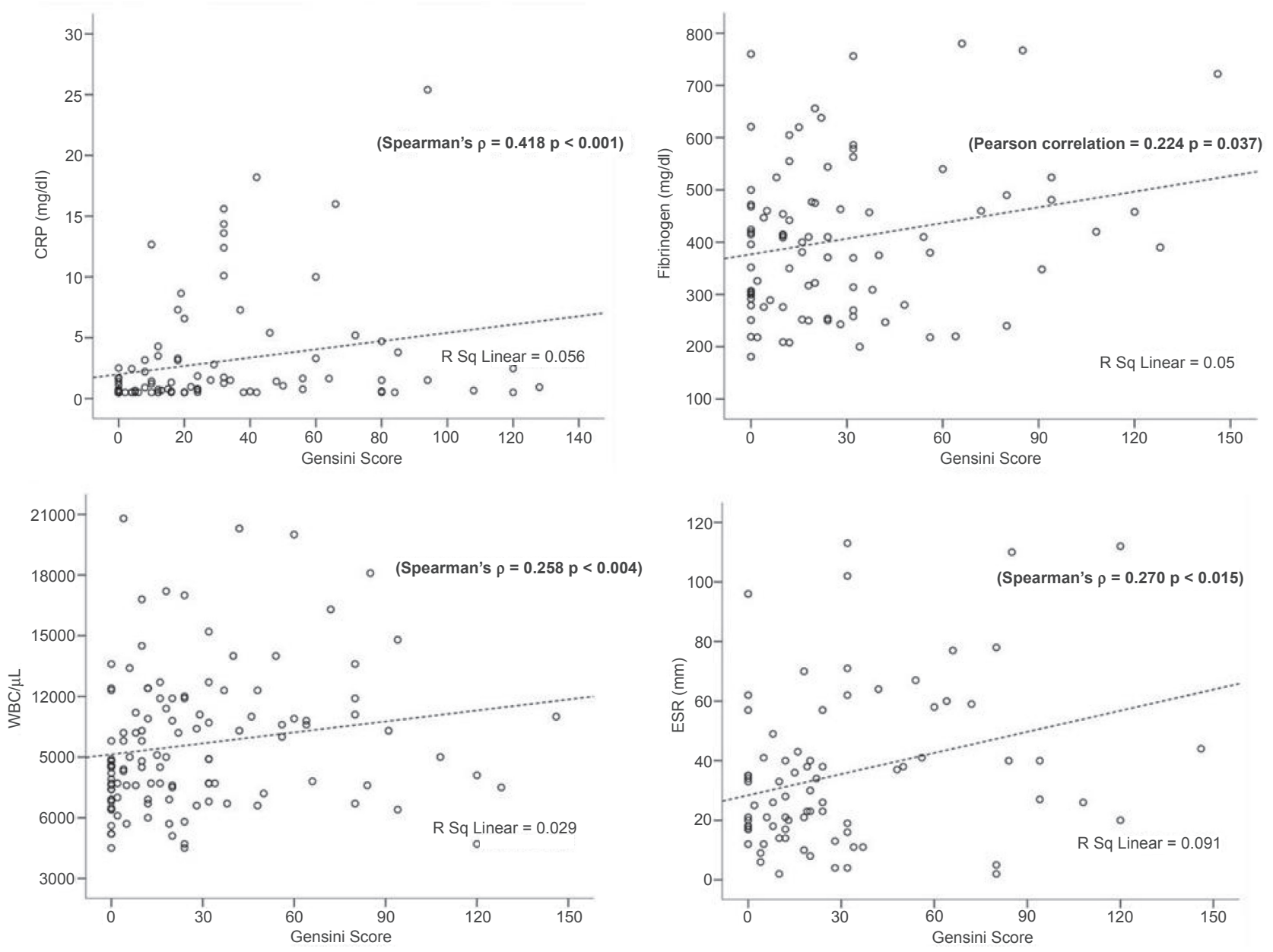

Figure 3 Scatter plots of peak serum CRP (mg/d)), fibrinogen $(\mathrm{mg} / \mathrm{dl})$, peripheral WBC count $(/ \mu \mathrm{L})$, and ESR $(\mathrm{mm} / \mathrm{h})$ with Gensini score in the whole study sample. Significant positive correlations between inflammatory biomarkers and Gensini score were identified.

In our study, the prevalence of arterial hypertension was also significantly higher among patients with acute, firstever MI and CAD without MI, in comparison with controls $(p=0.048)$. Prospective longitudinal analysis of follow-up data from the Framingham Study of the relation of antecedent blood pressure to occurrence of subsequent cardiovascular morbidity and mortality depending on the metabolically linked burden of associated risk factors has shown that hypertension is one of the most prevalent and powerful contributors to cardiovascular diseases (Kannel 1996; Vasan et al 2002).

Hyperlipidemia was noticed in higher proportion in the first two study groups in contrast with controls. A highly significant difference in the prevalence of hyperlipidemia was noted, especially in patients with acute MI $(\mathrm{p}<0.001)$. Hyperlipidemia is considered to be among the major cardiovascular risk factors. The role of serum cholesterol in predicting first coronary events is well established and reduction of serum cholesterol decreases the risk of CAD (Castelli et al 1986; Martin et al 1986; Anderson et al 1987; Pekkanen et al 1990; Law et al 1994a, 1994b; Gotto Jr 1998).
A strong association of positive family history with evidence of CAD was observed in our study. Patients with acute MI, had an increased prevalence of family history of cardiovascular disease in comparison with the other patient groups $(p=0.023)$. An early history of parental MI (less than 60 years of age) confers a greater risk of cardiovascular disease than does MI at older ages (Colditz et al 1991; LloydJones et al 2004).

Diabetes mellitus, a major cardiovascular risk factor was present in higher proportion of patients with acute MI and CAD without MI, in comparison with patients in the control group, but the difference observed was not significant in our sample $(p=0.134)$. Diabetes mellitus constitutes a major risk factor for cardiovascular events. Based on 20 years of surveillance of the Framingham cohort, which related subsequent cardiovascular events to prior evidence of diabetes, a 2-3-fold increased risk of clinical atherosclerotic disease including coronary heart disease, peripheral arterial disease, and congestive heart failure was reported (Kannel and McGee 1979; Fox et al 2007). 

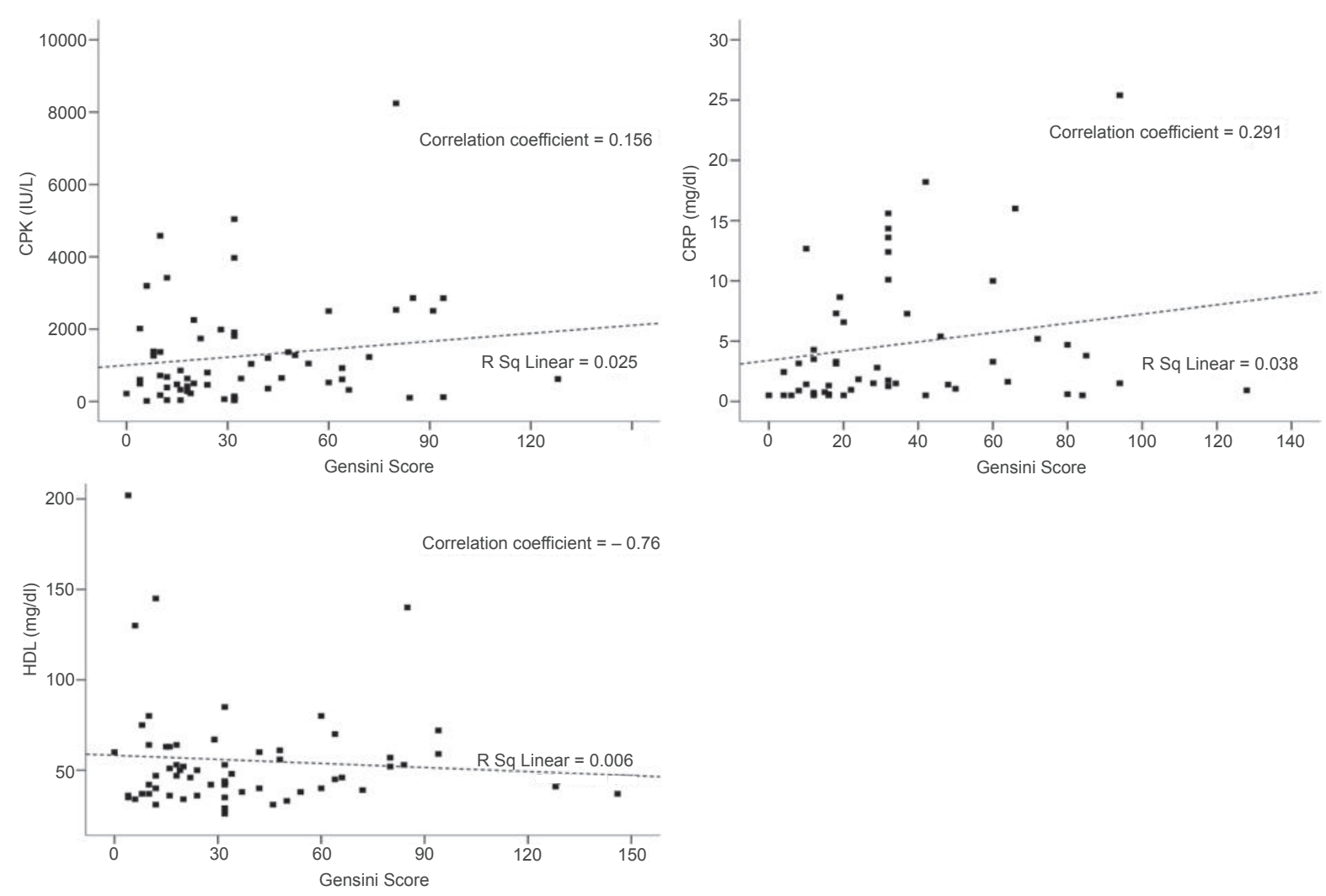

Figure 4 Scatter plots of peak serum CPK (IU/L), CRP ( $\mathrm{mg} / \mathrm{d})$ ), and HDL (mg/dl) with Gensini score in patients with acute, first ever MI. In this subgroup of patients, positive correlations between peak serum CPK and CRP with Gensini score were determined. Negative correlation between serum HDL and Gensini score was also identified.
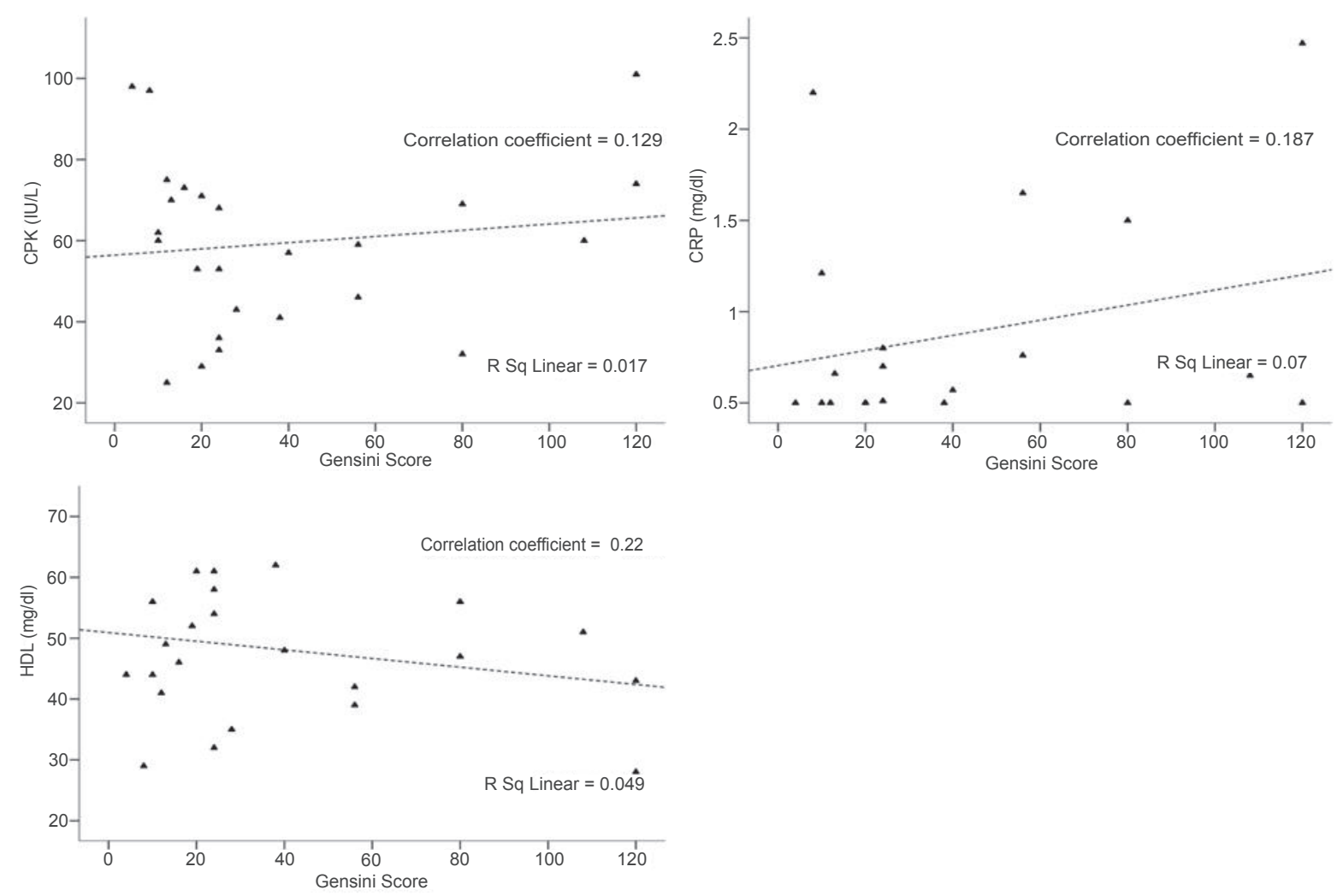

Figure 5 Scatter plots of peak serum CPK (IU/L), CRP (mg/dl), and HDL (mg/dl) with Gensini score in patients with newly-diagnosed CAD without MI. In this subgroup of patients, positive correlations between peak serum CPK and CRP with Gensini score were determined. Negative correlation between serum HDL and Gensini score was also identified. 
Hyperuricemia (serum uric acid levels $>7 \mathrm{mg} / \mathrm{dl}$ ) was present in higher proportion in patients with acute, firstever MI and patients with CAD without $\mathrm{MI}$ in comparison with controls. A difference was noticed, yet it did not yield statistical significance in our study sample $(p=0.676)$. Evidently, there seems to be an association between serum uric acid levels and CAD, since data from Health Professionals Follow-Up Study indicate that men with gout have a higher risk of death from all causes. Among men without preexisting $\mathrm{CAD}$, the increased mortality risk is primarily a result of an elevated risk of cardiovascular death, particularly from CAD (Choi and Curhan 2007).

The process of myocardial tissue necrosis is accompanied by elevations in enzyme serum levels of CPK, AST, LDH, and troponin due to myocardial cellular damage. Cardiac troponin (cTn) has established itself firmly as the "gold standard" in the diagnosis of acute coronary syndrome and is measured in all patients presenting with symptoms suggestive of acute coronary syndrome, in conjunction with physical examination and ECG. Because of the specificity of cTn for myocardial damage, a single cTn above the decision limit, along with clinical evidence, is indicative of myocardial injury (Möckel et al 1998; Panteghini 2004; Antman et al 2004; Melanson et al 2007; Morrow et al 2007). The latter holds true regarding our study, where mean levels of peak serum CPK, AST, and LDH were significantly higher in patients with acute first-ever MI compared with patients with CAD without MI and controls ( $\mathrm{p}<0.001)$. Most important was the demonstration of positive linear correlation between peak serum CPK, AST, and LDH with Gensini score (Spearman's $\rho=0.389, p<0.001$; Spearman's $\rho=0.391, p<0.001$; and Spearman's $\rho=0.366, p<0.001$, respectively). Therefore, higher myocardial enzyme peak serum levels were related to increased severity of CAD, represented by Gensini score.

Tissue necrosis is also the initiating event of a subsequent inflammatory process that accounts for a significant increase in serum inflammatory markers (CRP, fibrinogen, ESR, and peripheral WBC count) noticed in patients with acute MI. In our study, the mean value of CRP was significantly higher in patients with acute MI than in patients with CAD without MI and controls $(\mathrm{p}<0.001)$. CRP values correlated positively with CAD severity estimated by Gensini score (Spearman's $\rho=0.418, p<0.001)$. In consideration of the important role that inflammatory processes play in determining plaque stability, recent work has focused on whether plasma markers of inflammation may help improve risk stratification. Of these markers, CRP has been the most widely studied, and there is now robust evidence that CRP is a strong predictor of cardiovascular risk among apparently healthy individuals, patients undergoing elective revascularization procedures, and patients presenting with acute coronary syndromes (Berk et al 1990; Neumann et al 1995; Mendall et al 2000; Sepulveda and Mehta 2005; Brunetti et al 2006; Blake and Ridker 2007).

Inflammation is a recognized key component of acute coronary syndromes. Such pathogenetic achievement has led to the use of inflammatory cells and proteins as prognostic markers in these syndromes. A number of markers have been proposed, including proinflammatory cytokines such as interleukin-6, interleukin-1, and tumor necrosis factor- $\alpha$, adhesion molecules such as intracellular adhesion molecule- 1 and vascular adhesion molecule- 1 and markers of cell activation. Although all are of scientific interest, the clinical use of these markers is limited by their high cost, low availability, and unfavorable biological profile. Circulating levels of CRP rise during the acute-phase response to tissue injury, infection, and inflammation (Berk et al 1990; Danesh et al 1999; Mendall et al 2000; Biasucci et al 2004; Sepulveda and Mehta 2005; Brunetti et al 2006; Blake and Ridker 2007). Conversely, CRP, the prototypic acute phase protein, and to a lesser extent fibrinogen, have been proven to be reliable and important markers of risk in ischemic heart disease. CRP has been consistently found to be independent from other risk factors and to have an incremental value beyond the common risk factors and biochemical markers of risk, including troponin (Berk et al 1990; Danesh et al 1999; Mendall et al 2000; Biasucci et al 2004; Sepulveda and Mehta 2005; Brunetti et al 2006; Blake and Ridker 2007). CRP has been shown to exert proatherogenic effects on vascular cells exemplified by increasing the secretion of monocyte chemoattractant protein (Pasceri et al 2001), reducing nitric oxide bioactivity (Venugopagal et al 2002), and inducing adhesion molecules, such as vascular cell adhesion molecule-1, intercellular adhesion molecule-1, and E-selectin (Pasceri 2000).

Vascular inflammation can be limited by antiinflammatory counterregulatory mechanisms that maintain the integrity and homeostasis of the vasculature (Tedgui and Mallat 2001). HDL encompasses potent antiinflammatory properties which could account for the protective effect against atherogenesis (Cockerill et al 1995). Epidemiological and clinical studies showed that HDL concentration is often inversely correlated with the plasma levels of CRP, in atherosclerotic cardiovascular diseases (Ridker et al 2000). HDL molecules have an established role in the regression 
processes of atherosclerosis as well as a putative role as antiinflammatory agents. Elevation of CRP values in familial hypoalphalipoproteinemia, in the absence of signs and symptoms of local or systemic inflammation or systemic or recurrent disease, may suggest an upregulation of proinflammatory mechanisms, which is further exacerbated by the presence of coronary atherosclerotic disease (Sampietro et al 2002). Furthermore, among subjects with a visible lesion or stenosis in coronary arteries, those with low HDL cholesterol or smokers are more likely to develop MI (Sun et al 2006).

In our study, a significant reciprocal correlation between serum HDL and CRP and between serum HDL and Gensini score was demonstrated indicating a possible causal effect. These observations concerning HDL, Gensini score, and CRP merit further investigation in order to distinguish between statistical epiphenomena and possible causal relation.

Wadham and colleagues (2004) support that HDL via oxidation of its principal phospholipid neutralizes the proinflammatory potential of CRP in endothelial cells, revealing a balance between antiinflammatory and proinflammatory actions within the vascular wall. Factors that decrease the quantity and/or quality of HDL, such as obesity, diabetes, and age, are often associated with increased CRP concentration (Chambers et al 2001; Wadham et al 2004), which could ultimately disturb the antiinflammatory and proinflammatory balance to contribute to the development of inflammatory cardiovascular diseases. In a prospective study by Tarchalski and colleagues (2003) in angina pectoris patients with no previous MI, severity of coronary atherosclerosis was positively correlated with total cholesterol, LDL cholesterol, and triglycerides and negatively correlated with HDL cholesterol. In our study a negative association between serum HDL and Gensini score, the index of CAD severity is also established in patients with CAD without infarction, in patients with acute MI and controls.

Fibrinogen levels were also higher in patients with acute MI compared to other groups, yet the difference did not yield statistical significance in our sample $(p=0.14)$. Significant correlation was demonstrated regarding fibrinogen levels and Gensini score (Pearson correlation $=0.224, \mathrm{p}=0.037$ ). Fibrinogen levels play a critical role in both blood clot formation and vascular wall inflammation while genetic heterogeinity between individuals further determines susceptibility to CAD (Mannila 2006; Omran and Asadollahi 2007). Serum fibrinogen is also related to CAD and considered to be an independent predictor of acute MI. Fibrinogen was demonstrated, more than 40 years ago, to be elevated among patients with acute thrombosis (Eidelman and Hennekens 2003). The first prospective study to show an association between fibrinogen levels and subsequent cardiovascular disease risk was the Gothenburg Heart Study from Sweden in 1984 (Wilhelmsen et al 1984). In the Northwick Park Heart Study from the UK (Meade et al 1986), fibrinogen and factor VII appeared to be as effective as total cholesterol in predicting future risk of coronary heart disease. In the European Concerted Action on Thrombosis and Disabilities Angina Pectoris Study (Ross 1999), higher levels of fibrinogen predicted subsequent acute coronary syndromes while lower levels, despite elevated cholesterol levels, were associated with lower risks of acute coronary syndromes.

In our study mean values peripheral WBC counts were significantly higher in patients with acute MI than in patients with CAD without MI and controls ( $p<0.001$ ). Significant correlation was found regarding WBC count and Gensini score (Spearman's $\rho=0.258 \mathrm{p}=0.004$ ), indicating a strong association of leukocytosis with CAD severity, especially in patients with acute MI. Correlation of the leukocyte count with CAD and investigations into the utility of the leukocyte count as a risk factor and prognostic indicator in patients with CAD are consistent with the current concept that atherosclerosis is an inflammatory disease (Pearson et al 2003; Pai et al 2004; Madjid et al 2004; Jia et al 2005). Leukocytosis affects CAD through multiple pathologic mechanisms that mediate inflammation, cause proteolytic and oxidative damage to the endothelial cells, plug the microvasculature, induce hypercoagulability, and promote infarct expansion. In summary, leukocytosis has been consistently shown to be an independent risk factor and prognostic indicator of future cardiovascular outcomes, regardless of disease status. The leukocyte count is inexpensive, reliable, easy to interpret, and ordered routinely in inpatient and outpatient settings (Madjid et al 2004). The association of WBC count with the angiographical characteristics of coronary atherosclerosis, as estimated by Gensini score, strongly suggests that granulocytosis may play a role in the development of coronary atherosclerosis (Jia et al 2005).

ESR values were also higher in patients with acute MI compared to other groups, but this difference did not yield statistical significance in our sample $(p=0.33)$. However significant correlation regarding ESR and Gensini score was demonstrated, indicating the importance of ESR values in patients with severe CAD (Spearman's $\rho=0.270, p=0.015$ ). In 1988 the International Committee for Standardization in Hematology suggested that the ESR might be a particularly robust 
and appropriate test for monitoring chronic inflammatory processes since it is sensitive to both fibrinogen and immunoglobulins (ICSH 1988). Accordingly, a fast and inexpensive ESR test might be a valuable tool for assessing the strength of the inflammatory response associated with atherosclerosis, and might conceivably carry important short- and long-term prognostic information (Erikssen et al 2000). The ESR is a strong predictor of coronary heart disease mortality, and appears to be a marker of aggressive forms of coronary heart disease. The ESR probably gives substantial information in addition to that given by fibrinogen on the risk of coronary heart disease death (Erikssen and Mundal 1982).

\section{Study limitations}

A major limitation of our study was the selection of "control" patients. Although all patients in this group were subjected to coronary angiography that did not reveal critical coronary vessel stenoses the majority had underlying cardiovascular disease including dilated cardiomyopathy, valvular disorders, etc. Therefore, although a negative coronary angiogram eliminated the possibility of coexisting CAD, these subjects had some form of cardiological condition rendering their recruitment in the control group problematic. For practical reasons and since the main criterion for patient recruitment into study groups was the presence or absence of CAD, we regarded these patients as "controls," based in the absence of CAD, according to angiographic findings.

\section{Conclusions}

The objective of our study was to identify correlations between serum levels of myocardial enzymes, serum lipids, and inflammatory biomarkers, with CAD severity reflected by Gensini score, in Greek patients submitted to coronary angiography.

Significant positive correlations between angiographic findings, peak serum myocardial enzyme levels, and inflammatory biomarkers were identified. Myocardial enzyme serum levels and inflammatory biomarkers not only increased in the setting of an acute coronary event, but there was also a quantitative correlation with the extent of myocardial damage and CAD severity, according to angiographic findings (Gensini score). In this study, a reciprocal association between HDL levels and CAD severity (Gensini score) was established and an inverse correlation between serum HDL and CRP levels was demonstrated.

The correlation between peak serum myocardial enzyme levels (CPK, AST, LDH), markers of inflammation (CRP, fibrinogen, ESR, WBC) and HDL with severity of CAD evaluated by Gensini score shown in our study, could also convey important prognostic significance regarding the clinical setting. Evidence from our study demonstrated that simple biochemical assays provide an adequate estimation of CAD severity due to their correlation with Gensini score in hospitalized patients. The latter holds true, especially in patients submitted to coronary angiography due to acute MI. Serum enzyme levels and inflammatory indexes could be used to obtain a comprehensive view of the infarct size and severity of vascular stenotic lesions.

\section{Disclosure}

The authors report no conflicts of interest in this work.

\section{References}

Anderson KM, Castelli WP, Levy D. 1987. Cholesterol and mortality. 30 years of follow-up from the Framingham study. JAMA, 257:2176-80.

Antman EM, Anbe DT, Armstrong PW, et al. for the American College of Cardiology; American Heart Association Task Force on Practice Guidelines; Canadian Cardiovascular Society. 2004. ACC/AHA guidelines for the management of patients with ST-elevation myocardial infarction: a report of the American College of Cardiology/American Heart Association Task Force on Practice Guidelines (Committee to Revise the 1999 Guidelines for the Management of Patients with Acute Myocardial Infarction). Circulation, 110:e82-e293.

Berk BC, Weintraub WS, Alexander RW. 1990. Elevation of C-reactive protein in "active" coronary artery disease. Am J Cardiol, 65:168-72.

Biasucci LM, CDC, AHA. 2004. CDC/AHA Workshop on Markers of Inflammation and Cardiovascular Disease: Application to Clinical and Public Health Practice: clinical use of inflammatory markers in patients with cardiovascular diseases: a background paper. Circulation, 110:e560-e567.

Blake GJ, Ridker PM. 2003. C-reactive protein and other inflammatory risk markers in acute coronary syndromes. $\mathrm{J} \mathrm{Am} \mathrm{Coll} \mathrm{Cardiol,}$ 41(4 Suppl S):37S-42S

Brunetti ND, Troccoli R, Correale M, et al. 2006. C-reactive protein in patients with acute coronary syndrome: correlation with diagnosis, myocardial damage, ejection fraction and angiographic findings. Int J Cardiol, 109:248-56.

Castelli WP, Garrison RJ, Wilson PW, et al. 1986. Incidence of coronary heart disease and lipoprotein cholesterol levels. The Framingham Study. JAMA, 256:2835-8.

Chambers JC, Eda S, Bassett P, et al. 2001. C-reactive protein, insulin resistance, central obesity, and coronary heart disease risk in Indian Asians from the United Kingdom compared with European whites. Circulation, 104:145-50.

Choi HK, Curhan G. 2007. Independent impact of gout on mortality and risk for coronary heart disease. Circulation, 116:894-900.

Cockerill GW, Rye KA, Gamble JR, et al. 1995. High-density lipoproteins inhibit cytokine-induced expression of endothelial cell adhesion molecules. Arterioscler Thromb Vasc Biol, 15:1987-94.

Colditz GA, Rimm EB, Giovannucci E, et al. 1991. A prospective study of parental history of myocardial infarction and coronary artery disease in men. Am J Cardiol, 67:933-8

Conti CR. 1977. Coronary arteriography. Circulation, 55:227-37.

Danesh J, Muir J, Wong Y, et al. 1999. Risk factors for coronary heart disease and acute-phase proteins: A population-based study. Eur Heart $J$, 20:954-9.

Eidelman RS, Hennekens CH. 2003. Fibrinogen: a predictor of stroke and marker of atherosclerosis. Eur Heart J, 24:499-500. 
Erikssen G, Liestol K, Bjornholt JV, et al. 2000. Erythrocyte sedimentation rate: a possible marker of atherosclerosis and a strong predictor of coronary heart disease mortality. Eur Heart J, 21:1614-20.

Erikssen J, Mundal R. 1982. The non-infarct patient with coronary artery disease: can a high risk group be identified? Ann NY Acad Sci, 382:438-9.

Fox CS, Coady S, Sorlie PD, et al. 2007. Increasing cardiovascular disease burden due to diabetes mellitus. The Framingham Heart Study. Circulation, 115:1544-50.

Gensini GG. 1980. Chapter 10: Coronary arteriography. Pp. 308-62. In: Braunwald E (ed). Heart Disease. Philadelphia: WB Saunders Company.

Gensini GG. 1983. A more meaningful scoring system for determining the severity of coronary heart disease. Am J Cardiol, 51:606.

Gotto AM Jr. 1998. Triglyceride as a risk factor for coronary artery disease. Am J Cardiol, 82(9A):22Q-25Q.

Hayashi M, Fujimoto K, Urushibata K, et al. 2003. Nocturnal oxygen desaturation correlates with the severity of coronary atherosclerosis in coronary artery disease. Chest, 124:936-41.

[ICSH] International Committee for Standardization in Hematology (Expert Panel on Blood Rheology). 1988. Guidelines on selection of laboratory tests for monitoring the acute phase response. J Clin Pathol, 41:1203-12.

Jenkins DC, Rosenman RH, Zyzanski SJ. 1968. Cigarette smoking: Its relationship to coronary heart disease and related risk factors in the Western Collaborative Group Study. Circulation, 38:1140-55.

Jia EZ, Yang ZJ, Yuan B, et al. 2005. Relationship between leukocyte count and angiographical characteristics of coronary atherosclerosis. Acta Pharmacologica Sinica, 26:1057-62.

Kannel WB. 1996. Blood pressure as a cardiovascular risk factor: prevention and treatment. JAMA, 275:1571-6.

Kannel WB, McGee DL. 1979. Diabetes and cardiovascular disease. The Framingham study. JAMA, 241:2035-8.

Law MR, Wald NJ, Wu T, et al. 1994a. Systematic underestimation of association between serum cholesterol concentration and ischemic heart disease in observational studies: data from the BUPA study. $B M J, 308: 363-6$.

Law MR, Wald NJ, Thompson SG. 1994b. By how much and how quickly does reduction in serum cholesterol concentration lower risk of ischemic heart disease. BMJ, 308:367-73.

Lloyd-Jones DM, Nam BH, D’Agostino RB Sr, et al. 2004. Parental cardiovascular disease as a risk factor for cardiovascular disease in middle-aged adults. A prospective study of parents and offspring. JAMA, 291:2204-11.

Madjid M, Awan I, Willerson JT, et al. 2004. Leukocyte count and coronary heart disease: implications for risk assessment. J Am Coll Cardiol, 44:1945-56.

Mannila MN. 2006. Fibrinogen and susceptibility to myocardial infarction. Stockholm: Karolinska University Press.

Martin MJ, Hulley SB, Browner WS, et al. 1986. Serum cholesterol, blood pressure, and mortality: implications from a cohort of 361,662 men. Lancet, 2(8513):933-6.

Meade TO, Mellows S, Brozovic M, et al. 1986. Haemostatic function and ischaemic heart disease: principal results of the Northwick Park Heart Study. Lancet, 2(8506):533-7.

Melanson SE, Tanasijevic MJ, Jarolim P. et al. 2007. Cardiac troponin assays: a view from the clinical chemistry laboratory. Circulation, 116:e501-4.

Mendall MA, Strachan DP, Butland BK, et al. 2000. C-reactive protein: relation to total mortality, cardiovascular mortality and cardiovascular risk factors in men. Eur Heart J, 21:1584-90.

Möckel M, Störk T, Heller G Jr, et al. 1998. Troponin T in patients with low grade or atypical Angina. Identification of a high risk group for shortand long-term cardiovascular events. Eur Heart J, 19:1802-7.

Morrow DA, Cannon CP, Jesse RL, et al. 2007. National Academy of Clinical Biochemistry. National Academy of Clinical Biochemistry Laboratory Medicine Practice Guidelines: clinical characteristics and utilization of biochemical markers in acute coronary syndromes. Clin Chem, 53:552-74.
Mosca L, Collins P, Herrington DM, et al. 1997. American Heart Association. Cardiovascular disease in women. A statement for healthcare professionals from the American Heart Association. Circulation, 96:2468-82.

Neumann FJ, Ott I, Gawaz M, et al. 1995. Cardiac release of cytokines and inflammatory responses in acute myocardial infarction. Circulation, 92:748-55.

Omran MT, Asadollahi S. 2007. The measurement of serum fibrinogen levels in patients with acute coronary syndrome. Saudi Med J, 28:1350-2.

Pai J, Pischon T, Ma J, et al. 2004. Inflammatory markers and the risk of coronary heart disease in men and women. $N$ Engl J Med, 351:2599-610.

Panteghini M. 2004. Role and importance of biochemical markers in clinical cardiology. Eur Heart J, 25:1187-96.

Pasceri V, Willerson JT, Yeh ET. 2000. Direct proinflammatory effect of C-reactive protein on human endothelial cells. Circulation, 102:2165-68.

Pasceri V, Cheng JS, Willerson JT, et al. 2001. Modulation of C-reactive protein-mediated monocyte chemoattractant protein-1 induction in human endothelial cells by anti-atherosclerosis drugs. Circulation, 103:2531-4.

Pearson TA, Mensah GA, Alexander RW, et al.2003. Centers for Disease Control and Prevention; American Heart Association. Markers of inflammation and cardiovascular disease: application to clinical and public health practice: A statement for healthcare professionals from the centers for disease control and prevention and the American Heart Association. Circulation, 107:499-511.

Pekkanen J, Linn S, Heiss G, et al. 1990. Ten-year mortality from cardiovascular disease in relation to cholesterol level among men with and without preexisting cardiovascular disease. $N$ Engl J Med, 322:1700-7.

Ridker PM, Hennekens CH, Buring JE, et al. 2000. C-reactive protein and other markers of inflammation in the prediction of cardiovascular disease in women. $N$ Engl J Med, 342:836-43.

Ross R. 1999. Atherosclerosis - an inflammatory disease. N Engl J Med, 340:115-26.

Sampietro T, Bigazzi F, Dal Pino B, et al. 2002. Increased plasma C-reactive protein in familial hypoalphalipoproteinemia. A proinflammatory condition? Circulation, 105:11-4.

Scanlon PJ, Faxon DP, Audet AM, et al. 1999. ACC/AHA guidelines for coronary angiography. A report of the American College of Cardiology/ American Heart Association Task Force on practice guidelines (Committee on Coronary Angiography). Developed in collaboration with the Society for Cardiac Angiography and Interventions. $J$ Am Coll Cardiol, 33:1756-824.

Sepulveda JL, Mehta JL. 2005. C-reactive protein and cardiovascular disease: a critical appraisal. Curr Opin Cardiol, 20:407-16.

Sun YH, Yang YJ, Pei WD, et al. 2006. Patients with low high-density lipoprotein-cholesterol or smoking are more likely to develop myocardial infarction among subjects with a visible lesion or stenosis in coronary artery. Circ J, 70:1602-5.

Tarchalski J, Guzik P, Wysocki H. 2003. Correlation between the extent of coronary atherosclerosis and lipid profile. Mol Cell Biochem, 246:25-30.

Tedgui A, Mallat Z. 2001. Anti-inflammatory mechanisms in the vascular wall. Circ Res, 88:877-87.

Vasan RS, Massaro JM, Wilson PWF, et al. 2002. Antecedent blood pressure and risk of cardiovascular disease. The Framingham Heart Study. Circulation, 105:48-53.

Venugopal S.K. Devaraj S, Yuhanna I, et al. 2002. Demonstration that C-reactive protein decreases eNOS expression and bioactivity in human aortic endothelial cells. Circulation, 106:1439-41.

Wadham C, Albanese N, Roberts J, et al. 2004. High-density lipoproteins neutralize C-reactive protein proinflammatory activity. Circulation, 109:2116-22.

Wilhelmsen L, Svärdsudd K, Korsan-Bengtsen K, et al. 1984. Fibrinogen as a risk factor for stroke and myocardial infarction. N Engl J Med, 311:501-5.

Yasue H, Hirai N, Mizuno Y, et al. 2006. Low-grade inflammation, thrombogenicity, and atherogenic lipid profile in cigarette smokers. Circ J, 70:8-13. 